\title{
BJOG-20-2493.R1 A Biomarker for Amniotic Fluid Embolism: The Search Continues
}

\author{
Steven Clark ${ }^{1}$ \\ ${ }^{1}$ Baylor College of Medicine/Texas Childrens Hospital
}

May 21, 2021

Mini-Commentary on Manuscript \# BJOG-20-2493

A Biomarker for Amniotic Fluid Embolism: The Search Continues

Word count: 495

Research into the pregnancy associated cytokine storm-like condition historically known as amniotic fluid embolism (AFE) has been hampered by a lack of unique diagnostic criteria. In its classic form, the clinical presentation of this condition is unmistakable. In less classic presentations, each of the clinical hallmarks of AFE (depressed ventricular function, lung injury and coagulopathy) may, in isolation, be seen in other obstetric conditions. Indeed, much of the confusion arising from previously published case series purporting to describe women with AFE appears to be the result of the inclusion of patients with other conditions. (Clark SL et al Obstet Gynecol 2014:123: 337-48) Identification of a reliable, objective biomarker specific to AFE is badly needed.

It is against this background that the work of Bouvet et al is especially welcome. These investigators examined the use of insulin-like growth factor binding protein -1, a protein found in high concentration in amniotic fluid, as a potential biomarker of AFE in women suspected to have this condition. Unfortunately, the results were negative, leading the authors to question the usefulness of this assay.

Although the results were negative, the major importance of this study may be as an example of how to properly conduct a search for AFE biomarkers. These authors avoided several pitfalls that have invalidated most previous biomarker publications. First, they used 2 objective, internationally recognized clinical criteria sets for identifying women with AFE. Their finding that only about half of women suspected to have AFE actually had the condition based on either of the identified criteria sets emphasizes the importance of insisting on inclusion criteria more stringent than "someone thought the patient had AFE," so common in current literature. Secondly, in their use of women with suspected AFE these investigators avoided another common pitfall in biomarker research, namely the use of normal pregnant women, rather than critically ill women as controls. Presumably the women without AFE had some other form of critical illness. This distinction is particularly important in investigating the potential of various inflammatory mediators as specific markers for AFE.

Finally, the authors' data support 2 additional important conclusions beyond the original intent of the paper. The finding that $100 \%$ of AFE patients identified by the SMFM criteria also met the UK diagnostic criteria serve as additional validation of the ability of the former criteria to reliably identify a group of women who, for research purposes, do have the disease, while excluding some others with less typical forms of AFE. (Clark et al, Am J Obstet Gynecol 2016:408-12.) Secondly, the authors' findings of no difference in levels of ILGFBP-1 in women with and without clinical AFE, despite high levels of this protein in amniotic fluid, supports the current belief that amniotic fluid per se is unrelated to the condition known as amniotic fluid embolism. 
It is generally accepted by the scientific community that AFE is unpreventable. It is hoped that additional quality research such as that of Bouvet et al may someday change this unfortunate fact. 